\title{
«Les règles du jeu» dans "L’Heptaméron" de Marguerite de Navarre
}

\section{Edgard Pich}

\section{(2) OpenEdition}

1 Journals

Édition électronique

URL : http://journals.openedition.org/studifrancesi/1043

DOI : 10.4000/studifrancesi. 1043

ISSN : 2421-5856

Éditeur

Rosenberg \& Sellier

\section{Édition imprimée}

Date de publication : 1 novembre 2014

Pagination : 533-542

ISSN : 0039-2944

\section{Référence électronique}

Edgard Pich, « "Les règles du jeu» dans "L'Heptaméron" de Marguerite de Navarre », Studi Francesi [En ligne], 174 (LVIII | III) | 2014, mis en ligne le 01 novembre 2014, consulté le 17 septembre 2020. URL : http://journals.openedition.org/studifrancesi/1043 ; DOI : https://doi.org/10.4000/studifrancesi.1043

\section{(c) (1)}

Studi Francesi è distribuita con Licenza Creative Commons Attribuzione - Non commerciale - Non opere derivate 4.0 Internazionale. 


\title{
DISCUSSIONI E COMUNICAZIONI
}

\section{"Les règles du jeu»" dans "L'Heptaméron" de Marguerite de Navarre}

\begin{abstract}
The purpose of this experimental research concerns the first thirty short stories (out of 72) in the Heptaméron by Marguerite de Navarre, from a structural and enunciative point of view. The starting hypothesis is that this work is a game and the aim is to elucidate some of the rules of the game. Consequently: analysis of the enunciative situations (the weather and its repercussions, social, religious and monastic systems) of the various speeches and speakers; connections between narratives (one speaker) and discourses (several speakers); between fiction and history; the big importance of stock phrases; systematics of the characters, when and how they may begin to speak. The analysis of the content is more difficult to formalize, and we begin to deal with it by advancing a lot of hypotheses about the way the stories are linked from a thematic point of view.
\end{abstract}

L'étude qui suit ne porte que sur les trois premières journées de l'Heptaméron, soit les nouvelles 1 à 30 , pp. 68 à 345 , soit 277 sur les 526 pages de texte de l'édition Cazauran, soit plus de la moitié du texte. Elle ne se donne donc pas pour but de traiter de façon exhaustive du problème annoncé; elle propose seulement une formalisation de ce problème: cette formalisation devra nécessairement être précisée, nuancée, corrigée pour tenir compte de la suite de l'œuvre et des changements qu'on pourra y relever par rapport aux schémas que nous avons essayé de dégager.

L'Heptaméron est construit sur une alternance conte ou nouvelle/devis. Dans le conte le narrateur est un personnage; dans le devis le narrateur est l'«auteur», du moins ce qu'on peut appeler ainsi pour faire l'impasse sur certains problèmes. Le récit est toujours à la troisième personne et le narrateur-devisant fait très rarement des commentaires; dans les devis «l'auteur» anonyme cède le plus souvent la parole aux narrateurs des nouvelles, qui passent ainsi de la troisième personne à la première. Le devis est un «récit de paroles», quasi exclusivement; la nouvelle est un récit d'actions, même si des discours, des déclarations (mais pas de vrais dialogues) y apparaissent assez souvent.

Cette structuration est plus complexe qu'il n'y parait. Entre le conte et le devis, il y a une conjonction/disjonction difficile à analyser, mais très forte. En effet, le narrateur du conte prend toujours la parole avant de commencer son récit et la garde toujours un certain temps après la nouvelle, au début du devis. La séparation forte entre devis et nouvelle (discours/récit) se trouve ainsi affaiblie par le fait que le locuteur de l'un est l'un des locuteurs de l'autre et que la séparation typographique forte entre nouvelle et devis, avec le résumé et le titre qui servent à démarquer les deux structures, à les opposer, est un artifice qui n'est pas totalement en accord avec les structures énonciatives. De ce point de vue, la nouvelle 3, qui commence par une première personne constitue un hapax, dans les trois premières journées du moins, et cet

(1) Sauf indication contraire, nous citons l'édition de Nicole Cazauran, Folio Classique, 2000. L'expression «règle du jeu» est employée par un grand nombre de commentateurs. Il faut sans doute la relativiser, comme on le verra plus loin. 
hapax montre la complexité, plus grande qu'il n'y paraît, des structures énonciatives de l'ouvrage.

Du point de vue du contenu le devis ne constitue pas un examen objectif de la nouvelle, l'affirmation d'un point de vue extérieur. En effet, les indications selon lesquelles le contenu des nouvelles est en rapport avec les sentiments qu'entretiennent les personnages-narrateurs les uns avec les autres sont multiples. L'impersonnalité formelle des nouvelles est donc mise à mal par les devis: cette impersonnalité n'apparaît pas comme un donné authentique, mais comme une fiction, voire un stratagème à la faveur duquel les personnages-narrateurs peuvent dire d'une façon symbolique, indirecte, ce qu'ils ne peuvent pas dire à la première personne ${ }^{2}$. C'est encore une convention, un jeu, mais qui ne trompe personne, à moins qu'il s'agisse d'une thérapie à la faveur de laquelle on pourrait gérer des situations personnelles, psychologiques ou morales ou religieuses ou sociales, difficiles, qui d'ailleurs ne sont jamais affrontées directement, mais seulement de façon allusive: dans ce cas, le lecteur en sait toujours moins que les devisants; alors que, fictivement, la nouvelle est censée tout dire sur les événements et les rapports entre les personnages. À certains égards, l'ouvrage est le récit d'une «retraite» (dans le sens religieux du terme): pour des raisons contingentes (météorologiques), les devisants trouvent un temps pour s'abstraire des affaires du monde, ou au moins de les mettre à distance (de façon relative cependant) pour penser à leur salut éternel.

On notera néanmoins que le mot «règle» doit être aussi pris dans un autre sens: nous sommes dans un monastère et les devisants obéissent à une règle plus générale qui s'applique à leur emploi du temps: le matin: leçon et messe; l'après midi: nouvelles et devis, vêpres. Oisille joue le rôle de prieure, et l'ouvrage que nous lisons est une sorte de bréviaire, avec une succession de textes qui obéissent à des règles de contenu et de forme bien plus stricts qu'on ne pourrait l'imaginer, avec toutefois une certaine liberté dans les contenus des propos - plutôt qu'une œuvre littéraire dans le sens moderne. En dépit des attaques très vives contre les moines, les laïcs devisants vivent malgré eux, pendant un certain temps, une vie qui a bien des caractéristiques de la vie monastique, au point d'ailleurs qu'il se produit une sorte d'osmose entre la vie des vrais moines (qui viennent en catimini d'abord, puis ouvertement écouter les nouvelles des devisants), et celle des devisants (qui assistent à une partie des offices monastiques, messe et vêpres, sans compter les véritables «matines» que leur ménage la dévote Oisille chaque matin).

D'une autre façon on peut dire que la structure binaire de l'ouvrage est une mise en rapport entre d'une part le récit d'une praxis fictive (ou passée, ce qui d'une certaine façon revient au même) c'est-à-dire qui n'existe qu'in absentia, conventionnellement éloignée, enregistrée, avec un télos (un achèvement) bien précisé - et d'autre part une praxis in praesentia, inachevée, qui engage les différents conteurs/devisants, dont le caractère évolutif et inachevé est posé en principe. Le langage a donc deux fonctions tout à fait différentes: d'un côté (devis), c'est un acte dont les tenants sont partiellement obscurs et les aboutissants pris dans une succession dont ni les devisants ni le lecteur n'ont la maîtrise; de l'autre (nouvelles), ce sont des faits, dont les tenants et les aboutissants sont a priori clairs et, comme dans la tragédie aristotélicienne «achevés» (au sens pragmatique et non grammatical du terme, puisque les nouvelles sont au passé simple et non au passé composé). Les récits d'actes (nouvelles) peuvent être soumis à une analyse narratologique (syntagmatique), alors que les récits de paroles (devis) peuvent être analysés selon une méthode paradigmatique

(2) «Je vois bien que Nomerfide veut parler, parquoi je lui donne ma voix afin qu'elle décharge son cœur sur quelque bonne nouvelle» dit Guébron, nouvelle 5, devis, p. 101. 
(quel type de nouvelle produit tel devisant? Quel type de réaction manifeste-t-il à l'écoute des récits des autres devisants?). Il y a là une lecture verticale du texte où on ne peut avancer qu'au milieu de beaucoup de difficultés ${ }^{3}$.

Un dernier point permet de se rendre compte de la complexité, voire de l'ambiguité de l'ensemble de l'ouvrage: c'est le caractère de «vérité», qui est attribué avec une insistance étonnante à toutes les histoires qui sont rapportées. À quoi il faut ajouter la présence de l'auteur dans les événements qui sont rapportés: le nombre des cas où le personnage historique de Marguerite de Navarre est témoin des faits, quand elle n'est pas elle-même une actrice, est tellement élevé, que l'ouvrage, d'une certaine façon peut être lu, non pas certes, comme une autobiographie (structure impensable dans le contexte idéologique de l'époque), mais comme des sortes de mémoires ${ }^{4}$. D'autre part le caractère fictif d'un groupe d'aristocrates retenus par hasard et se réunissant pour se raconter des nouvelles, est contredit par le passage du premier Prologue où cette fiction est présentée, non comme une réalité assurément, mais comme un projet sérieux et réel, qui n'a pu être réalisé pour des raisons contingentes, et dont les principaux personnages devaient être le roi François I ${ }^{\mathrm{er}}$, sa sœur Marguerite, le dauphin et la dauphine (pp. 65-66). Par l'une de ses structures les plus clairement affirmées, l'ouvrage est donc historique et le fait que les événements rapportés soient tous, sans exception, élevés à la dignité de l'«exemplum» ou du «cas» (casuistique) et relèvent de ce fait, comme il est normal, d'une analyse psychologique, morale, religieuse, renvoie peut-être à une conception de l'histoire qui nous est totalement étrangère, mais qu'on peut comprendre.

\section{Quelques exemples du caractère ritualisé, voire formulaire de l'écriture}

\section{Dans la nouvelle}

La première phrase de la narration commence toujours par un complément de lieu, plus rarement de temps (le «Il était une fois» du conte), ou les deux compléments à la suite (6).

Compléments de lieu à l'incipit: nouvelles $1,2,4,5,7,8,9,10,11,14,17,18$, $20,22,23,25,27,29$.

(3) Ce sujet, très épineux, a été traité de façon convaincante par A. TOURNON dans une communication Ignorant les premières causes», dans le n. 10 de la revue «Textuel», dont l'essentiel peut être résumé par la phrase suivante: «les indices d'énigmes disséminés dans L'Heptaméron ne désignent pas seulement les masques en usage dans la vie de cour; ils renvoient, bien au-delà de ces accessoires de la dissimulation, à l'incapacité radicale de chacun à connaitre la vérité sur les autres et peut-être sur lui-même» (p. 87). L'ouvrage de M. M. DE LA GARANDERIE, Le Dialogue des romanciers. Une nouvelle lecture de 'L'Heptaméron' (Minard, Lettres modernes, 1977) est très décevant. C'est une série de monographies, sur chacun des devisants, où les productions verbales et les traits personnels de chacun sont regroupés; la seule hypothèse avancée, c'est celle de «dix cycles», un pour chacun des devisants, au lieu d'une série de 100 nouvelles (p. 12). Voir aussi quelques remarques de P. JOURDA, dans Marguerite d'Angoulème, Genève, Slatkine Reprints, pp. 962-963. On pourrait, au prix d'un anachronisme difficile à justifier, penser à $\mathrm{La}$ Vie.
Mode d'emploi de G. Perec (1978) ou à Passage de Milan, premier roman de Butor (1954), ou encore à certains manuscrits arabes, qui peuvent se lire de droite à gauche et de haut en bas. Le modèle sur lequel est construite l'œuvre est évidemment celui du jeu d'échecs. Peut-être faudrait-il se référer aux jeux poétiques (pas narratifs) des Grands Rhétoriqueurs. Mais, pour l'instant, l'œuvre conserve au moins en partie son caractère énigmatique.

(4) Le fait que les devisants-narrateurs aient des noms fictifs, mais des référents réels, ou du moins qu'il existe des «clefs», outre le fait que ces noms «propres» ont une signification - par exemple Hircus, le bouc pourrait donner son nom au mari de Parlamente, Hircan, à une époque où tout le monde connaît le latin, même si le nom Hircan apparaît dans la Bible, même s'il peut être lu comme l'anagramme d'Henri, mari de Marguerite - confirme cette dimension de l'ouvrage: des «mémoires secrets», en somme. Lucien FEBVRE parle d' «histoires vraies romancées» (Amour sacré, Amour profane, 1944, repris en Folio, p. 345) 
Compléments de temps (nous considérons également comme compléments de temps les références à des personnages historiques, par exemple: «En la cour du Roi et Reine de Castille», 24): 6, 12, 13, 15, 16, 19, 21, 24, 26, 28, 30.

La nouvelle 3 constitue une exception remarquable: elle commence par un discours à la première personne du narrateur.

\section{Dans le devis}

C'est toujours le narrateur qui vient d'achever son récit qui prend la parole le premier après le blanc qui sépare la nouvelle du devis. La première phrase du devis comporte presque toujours le verbe voir ou voilà ou un quasi synonyme (montrer, il me semble), ainsi que le vocatif «mes dames» ${ }^{5}$. Ce vocatif, qui ne correspond pas à la réalité de la situation, souligne le recours à une écriture formulaire. De même l'expression «donner sa voix» à quelqu'un (exceptions: 6, 9, 19, 28, 29). Le caractère formulaire du devis est quelque peu inattendu puisque le devis est constitué par des propos tenus par des personnages supposés réels et en vie, donc qui pourraient parler dans une sorte d'improvisation. On attendrait davantage ces caractéristiques dans la partie narrative, comme dans l'épopée homérique ou la chanson de geste. Mais on peut imaginer qu'une analyse plus fine des nouvelles permettrait de mettre en évidence certains caractères formulaires dans le récit lui-même.

Autre manifestation du caractère formulaire du devis, le fait que les deux dernières répliques des devis sont presque systématiquement la première attribuée au narrateur de la nouvelle qui précède («Je vous donne ma voix»), la seconde au narrateur de la nouvelle qui suit, qui prononce une sorte de préface (exceptions: 10 et $30)$, de «pacte narratif», de serment, d'engagement: voici les règles du récit que vous allez entendre: je vais «montrer» ceci ou cela, dire «la vérité» (exceptions: 2, 3, 7, $10,11,13,15,16,18,21$, et 27 à 30 : mais ces exceptions seraient à examiner plus attentivement).

On peut juger (au moins du point de vue du lecteur moderne) que ce pacte de véridiction entre plus ou moins en concurrence avec le caractère démonstratif et exemplaire du conte, qui est exprimé de deux façons:

- $\quad$ par le verbe montrer et ses synonymes, qui est employé au début et/ou à la fin de

la nouvelle. Pour ne citer que l'emploi du verbe montrer: 3 (début), 7 (fin), 13

(fin), 15 (début), 19 (début), Prologue 2 (fin), 21 (début), 22 (début), 25 (fin), 26 (début), 27 (fin), 28 (fin);

- par le mot exemple (l'«exemplum» de la rhétorique classique) souvent au début du devis (effet de généralisation: 3 («une histoire que volontiers je vous montre ici par exemple»), 5, 6, 7, 9 10, 24.

\section{Relevé des séquences formulaires en début de devis}

1 «e vous supplie, mes dames, regardez quel mal il vient»

2 «Voilà, mes dames, une histoire véritable»

3 «Voilà, mes dames, une histoire»

4 «Voilà, mes dames, qui devrait donner grande crainte»

5 «e vous prie, mes dames, pensez que»

6 «Par ceci voyez-vous, mes dames, combien prompte et subtile»

7 «Par ceci, voyez-vous, mes dames, que la finesse d'un homme»

(5) Avec une seule exception, doublement remarquable, «messieurs», puisqu'elle est due à

Dagoussin, un homme. 
8 «ll me semble, mes dames que»

9 «Vous semble-t-il, messieurs qui n'avez voulu croire à ma parole»

10 «Je sais bien, mes dames, que cette longue histoire»

11 «Il me semble, mes dames, que ce conte»

12 «Voilà mes dames, qui doit bien vous faire craindre ce petit Dieu»

13 «l me semble, mes dames, que celles à qui on présente de telles choses»

14 «Il me semble, mes dames»

15 «Voilà, mes dames, que sans épargner notre sexe, j’ai bien voulu montrer aux maris»

16 «Et pour ce, mes dames, vous garderez»

17 «Quant à moi, mes dames, je ne vois point»

18 «Je vous prie, mes dames, trouvez-moi une femme»

19 «Vous ne pouvez ici ignorer, mes dames, que l'amour de l'homme ne se soit montrée la plus grande»

20 «Par quoi, mes dames, je vous supplie»

21 «Or, mes dames, je vous prie que les hommes qui nous veulent peindre tant inconstantes, viennent maintenant ici et me montrent»

22 «Voilà, mes dames, une histoire qui est bien pour montrer»

23 «Je crois, mes dames, qu'après avoir entendu cette histoire très véritable»

24 «Par exemple ne doit nul serviteur confesser ce qui lui peut nuire et en rien aider.

Et encore moins, mes dames»

25 «C'est afin que vous connaissiez, mes dames»

26 «Voilà, mes dames, la différence d'un sage à une folle dame»

27 «ll me semble, mes dames, que par ce conte»

28 (exception)

29 «Mes dames, le maître, s'il servait»

30 «oilà, mes dames, comme il en prend à celles»

Les «règles» que nous avons essayé de mettre en évidence, ainsi que le caractère formulaire du propos, excluent la notion de «style» dans l'analyse de l'œuvre: l'outil que manient tous les devisants à la fois dans les nouvelles et les devis, relève de la pure rhétorique $^{6}$ (adaptation du propos au sujet, au destinataire, à la situation); le récit met l'accent sur le pathos (caractère tragique, comique, facétieux, inattendu...) des événements racontés, dans leur rapport avec les personnages qui les vivent; le devis sur l'éthos: chaque devisant s'exprime avec son caractère, ses idée, conformément à son personnage; Hircan avec une violence virile; Oisille est une «anus» (vieille femme en latin); Nomerfide est une «puella», Gébron est un «vieux monsieur sympathique» (L. Febvre), un «senex», et les opinions de chacune sont en rapport avec sa «persona» (son rôle social compte tenu de son sexe, son origine, son âge, son statut...)

\section{Système des énonciateurs}

Pour chaque nouvelle nous donnons:

1 Le numéro d'ordre

2 Le nombre de pages ou parties de pages dans l'édition N. Cazauran

3 Le sexe du narrateur (M/F)

(6) Le prologue oppose la «vérité des nouvelles» à la «rhétorique»; en fait, la «vérité» dont il est question est la vérité du «dit»; le style est la vérité du «dire». Voir, par exemple, J. LECOINTE, 'L'Hep- taméron': les lieux rhétoriques de la personne, in Marguerite de Navarre, Mont-de-Marsan, Ed. Interuniversitaires, 1995, pp. 511-525. 
4 Le nom du narrateur en italiques

5 La liste de devisants, à laquelle il faut ajouter le nom du narrateur de la nouvelle considérée (n. 3 ci-dessus) et le nom du narrateur de la nouvelle suivante, les deux dans le n. 3, le premier sur la même ligne, le second sur la ligne suivante, puisque ces deux locuteurs interviennent chaque fois et dans le même ordre obligatoirement à la fin du devis.

Prologue Parlamente, Longarine, Emarsuitte, Nomerfide, Oisille, Hircan, Simontault

110 pp. M Simontault, Parlamente

24 pp. F Oisille

37 pp. M Saffredent

48 pp. F Emarsuitte, Hircan, Nomerfide

54 pp. M Guébron, Longarine, Oisille

62 pp. F Nomerfide, Parlamente

72 pp. M Hircan, Parlamente

85 pp. F Longarine, Saffredent, Hircan, Guébron, Simontault, Parlamente

96 pp. M Dagoucin, Hircan, Oisille, Saffredent

1036 pp. F Parlamente, Hircan, Oisille, Saffredent, Longarine, Guébron

Prologue Parlamente, Nomerfide

116 pp. F Nomerfide, Oisille

127 pp. M Dagoucin, Emarsuitte, Hircan, Simontault, Guébron, Saffredent, Longarine

1314 pp. F Parlamente, Nomerfide, Hircan, Oisille, Emarsuitte, Guébron, Saffredent

147 pp. M Simontault, Guébron, Emarsuitte, Parlemente, Nomerfide, Oisille, Hircan, Saffredent, Dagoucin

1515 pp. F Longarine, Hircan, Emarsuitte, Parlamente, Oisille, Saffredent, Nomerfide

165 pp. M Guébron, Hircan, Nomerfide, Saffredent

174 pp. F Oisille, Parlamente, Guébron, Emarsuitte

185 pp. M Hircan, Oisille, Guébron, Parlamente, Longarine, Simontault, Dagoucin, Saffredent, Nomerfide

1911 pp. F Emarsuitte, Hircan, Oisille, Guébron, Parlamente, Longarine, Simontault, Dagoucin

202 pp. M Saffredent, Oisille, Hircan, Simontault, Longarine, Parlamente, Guébron

Prologue Saffredent, Parlemente

2121 pp. F Parlamente, Oisille, Longarine, Hircan, Simontault

$2212 \mathrm{pp}$. M Guébron, Nomerfide, Emarsuitte

238 pp. F Oisille, Hircan, Guébron, Parlamente, Emarsuitte, Saffredent, Simontault

249 pp. M Dagoucin, Guébron, Parlamente, Hircan, Simontault, Oisille, Saffredent, Emarsuitte, Nomerfide

255 pp. F Longarine, Guébron, Oisille, Nomerfide, Parlamente, Hircan

2615 pp. M Saffredent, Oisille, Parlemente, Hircan, Nomerfide, Guébron, Longarine

272 pp. F Emarsuitte, Oisille

284 pp. M Simontault, Guébron, Parlemente

292 pp. F Nomerfide, Guébron, Parlamente, Saffredent

306 pp. M Hircan, Oisille, Parlemente, Guébron, Longarine, Emarsuitte, Saffredent. 


\section{Quelques remarques}

1. La longueur des nouvelles est très variable et par conséquent les différents narrateurs occupent des espaces très différents:

Parlamente: 71 pp. ou parties de page

Longarine: 25

Saffredent: 24

Dagoucin: 22

Simontault, Emarsuitte, Guébron: 21

Oisille: 16

Hircan: 13

Nomerfide: 10

2. La longueur des devis varie aussi mais de façon moins importante. Le nombre d'interventions des divers devisants, en plus des deux interventions canoniques, avant et après la nouvelle proprement dite, du narrateur en position de devisant, est au contraire très variable, comme le montre le relevé suivant:

Parlamente: intervient dans 17 devis

Hircan, Guébron et Oisille dans 16

Saffredent dans 12

Nomerfide dans 10

Longarine et Emarsuitte dans 9

Simontault dans 8

On notera que les deux relevés confirment sans contestation la place éminente occupée par Parlamente (la bavarde...); mais Hircan occupe des positions qui ne sont pas symétriques, et de même Simontault, mais d'une façon inverse. Enfin, on ne trouve que trois devis dans lesquels chacun des dix devisants interviennent $(14,18$, 24).

L'articulation des nouvelles et des devis semble pouvoir être décrite, au moins de façon partielle, de la façon suivante. Chaque devis est un pivot autour duquel est construite à la fois l'opposition et la succession des nouvelles. En d'autres termes, chaque nouvelle est suivie d'un devis qui a trois fonctions:

1. discuter la signification morale qu'a «montrée» la nouvelle qui précède: fonction morale, religieuse, avec, assez souvent un aspect polémique

2. ancrer le débat dans les rapports sentimentaux et idéologiques que les devisants entretiennent entre eux: fonction référentielle (référence fictive, a priori du moins)

3. «Donner la voix» à un nouveau conteur qui se propose de «montrer» une autre réalité humaine différente de voire opposée ${ }^{7}$ à celle qui était mise en scène dans la nouvelle précédente: fonction d'échange social: $\mathrm{A}$ donne la parole à $\mathrm{B}$, qui la donne à $\mathrm{C}$ etc.

Thématiquement on pourrait formaliser ainsi la succession des nouvelles, surtout dans la première journée: nouvelle 1 vs nouvelle 2 vs nouvelle 3 etc. De même la succession des narrateurs obéit à l'opposition entre le masculin et le féminin (cette fois sans exception). On pourrait imaginer d'autres systèmes d'opposition/articulation: jeune/âgé, éthique traditionnelle/éthique nouvelle etc.

(7) Adrien de Thou écrit le 8 août 1553: «la fin de la précédente [nouvelle] donne demi-intelli-

gence à la subséquente». 
Malgré les difficultés très nombreuses que présente cette entreprise, nous allons proposer une série d'hypothèse, probablement à réviser, en tout cas à préciser ${ }^{8}$ sur le système d'enchaînement des nouvelles à partir de la règle énoncée ci-dessus'. Mais il n'est pas évident que cet enchaînement adversatif ait été systématiquement recherché. Nous reproduisons également les prétendus résumés de l'ensemble des nouvelles de chaque journée qu'on trouve dans le manuscrit (1512) que reproduit dans son édition Gisèle Mathieu-Castellani, bien que ces résumés soient plus que contestables. Les systèmes d'opposition sur lesquels pourrait être fondée la succession des nouvelles, présentent encore une autre faiblesse, dans la mesure où ces oppositions ne sont pas nécessairement l'élément le plus important du point de vue de la narration.

Première journée: «Mauvais tours que les femmes ont faits aux hommes et les hommes aux femmes» (Ms. 1512).

1/2 Crimes suscités par une femme débauchée vs Une femme honnête et chaste

2/3 Tragique vs Comique

3/4 Double cocuage vs Prudence et chasteté féminine

4/5 Noblesse vs Peuple

5/6 Loyauté vs Ruse féminine punie

6/7 Dissimulation féminine vs Ruse masculine

7/8 Un adultère triomphant vs Un adultère cocufié

8/9 Désordres du désir vs Un «feu celé et couvert» aboutit à la mort

$9 / 10$ «Amour profane» vs «Amour sacré» (L. Fèbre) (nouvelle exceptionnelle par sa longueur, qui a de toute évidence une valeur conclusive: montée spirituelle à partir de la nouvelle 1)

Deuxième journée: «Ce qui promptement tombe en fantaisie d'un chacun». $11 / 12$ Un moine facétieux ou un conte scatologique $(\text { ? })^{10}$ vs Un épisode tragique dans l'histoire des Médicis (Florence)

12/13 Conflit entre l'honneur familial et le lien féodal à Florence vs Comment une femme honnête se débarrasse des importunités de son «serviteur».

13/14 Un «serviteur» qui sombre dans la mort parce qu'il est sincère vs À Milan, un soupirant éconduit prend au dernier moment la place d'un soupirant accepté, avec l'accord de la maîtresse.

14/15 Substitution d'un amant éconduit à un amant d'abord accepté vs Une femme jeune, belle et fidèle se venge cruellement d'un mari vieux, infidèle et jaloux.

15/16 Cruauté d'une femme à l'égard d'un mari jaloux vs Épreuves que fait subir une veuve à son amant, dont elle est éprise depuis toujours, au terme desquelles ils s'aiment pour toujours.

16/17 Nouvelle galante $v$ s Sens politique de François $I^{\mathrm{er}}$.

17/18 Sens politique de François $I^{\text {er }}$ vs Une femme adulte éprouve la loyauté de son très jeune amant mais va si loin qu'elle risque de le perdre (cf. nouvelle 16).

18/19 Jeux dangereux avec le corps vs Relations purement platoniques de deux amoureux séparés par leur condition sociale et qui entrent tous deux en religion.

19/20 Éternité de l'amour humain sublimé en amour divin vs Brutale rupture entre un gentilhomme découvrant la dame dont il est le serviteur en train de faire une partie de jambes en l'air avec un palefrenier.

(8) Le but est d'indiquer une méthode de travail et non de donner des acquis.

(9) On pourrait également se demander si le pays où se déroulent les événements (Espagne pour les nouvelles 10 et 24 , Italie pour les nouvelles 3, 12, $14 ; 16,19$, France pour les autres) ne joue pas un certain rôle.

(10) Le texte de cette nouvelle pose problème. 
Troisième journée: «Des dames qui en leur amitié n'ont cherché nulle fin que l'honnêtetée ${ }^{11}$, et de l'hypocrisie et méchanceté des religieux».

21/22 Un bâtard et une fille sérieuse arrivent à se marier secrètement et à communiquer chastement, malgré les obstacles qu'une reine leur oppose avec acharnement. Le père finit par emprisonner sa fille et son mari, ayant fui en Allemagne, y trouve une compagne. Réconciliation entre le père et la fille. Celle-ci épouse un homme honnête et ils deviennent riches. Vs Du monde aristocratique au monde ecclésiastique: un prieur tente sans succès d'abuser d'une religieuse.

22/23 Une honnête religieuse résiste victorieusement à un prieur lubrique vs Un cordelier arrive à séduire une femme honnête, causant ainsi la mort de la femme, de son mari et de leur enfant.

23/24 Méchanceté d'une reine (nouvelle 21) vs Une reine d'Espagne fait subir à son serviteur des épreuves d'une telle cruauté que celui-ci est délivré d'une passion aliénante au profit de l'amour céleste.

24/25 Une reine punie de la cruauté avec laquelle elle a traité son loyal serviteur $v s$ Pour rencontrer une maittresse, un roi se fait passer pour un grand dévot.

25/26 Les rois ont tous les droits $v s$ Lorsqu'un couple sans enfant adopte un garçon et que celui-ci et sa mère adoptive tombent amoureux, il ne reste plus à celle-ci, pour conserver son honneur, qu'à mourir.

26/27 Une femme honnête meurt de ne pas succomber à une passion honnête vs Une femme joue un mauvais tour, mais justifié, à un galant ridicule.

27/28 Un galant ridicule berné par la femme qu'il convoite $v s$ Un galant ridicule berné par un astucieux Gascon qu'il avait voulu faire chanter.

28/29 Un galant ridicule berné vs Un curé galant berne le stupide paysan qui est le mari de sa belle.

29/30 Un paysan stupide berné par sa femme et son curé vs Conséquences de la pruderie d'une femme: un double inceste: mère-fils, une fille épouse son père-frère: tragiques pour la femme, mais sans nulle conséquence pour ses deux enfants (conclusion évidente de la troisième journée).

\section{Les contenus idéologiques}

Les contenus idéologiques, comme on l'a vu dans les hypothèses proposées cidessus, jouent un rôle important dans la mise en œuvre de la «varietas» à la fois des nouvelles et des devis. Leur nature et leur importance ne pose pas de problème majeur, parce que, dans ce domaine, tout est attendu, convenu: on peut en trouver trois principaux.

1. Les oppositions entre sexes: mais il semble que dans ce domaine on ait beaucoup de lieux communs, plus qu'une pensée ferme.

2. Les relations entre sexes: ces relations semblent regroupées sous trois chapitres différents:

- le plaisir physique, dont la poursuite, aussi bien chez les hommes que les femmes se situe dans un contexte en proie à l'anarchie (relations adultères hors mariage, viols...). La brutalité, voire la «bestialité» (le mot est utilisé) de ces relations est évoquée sans pudeur excessive.

(11) Cette expression fait partie du «pacte» de la $21^{\circ}$ et première nouvelle de cette journée (Par- 
- la relation socialisée dans le cadre du mariage et la famille; les concepts de loi et surtout d' «honneur» (le regard extérieur, la bonne réputation, le «bruit»y jouent un rôle essentiel). C'est un fait, un donné social, qu'il est vain de contester, même si les discussions sont nombreuses à ce sujet. Bien que les propos soient parfois conventionnels, certaines formules sont d'une vigueur dans la protestation qui mérite l'attention, par exemple: «L'honneur des hommes et des femmes n'est pas semblable» (p. 325, entendez: la femme qui fait son mari cocu est mise au ban de la société; l'homme marié qui a une maîtresse est un «galant»); «Nos tentations ne sont pareilles aux vôtres» (p. 328, entendez: la femme cherche hors mariage une relation où elle soit respectée et libre; l'homme ne cherche qu'à exercer la supériorité qu'il s'attribue). De même, la façon dont un double inceste est présenté et absous en soi, sinon dans la personne responsable, une mère abusive, est d'une audace singulière.

- Le platonisme revu et corrigé par la vulgate pétrarquisante, qui se développe dans des relations, souvent appelées «amitiés», entre un «serviteur» et la femme aimée; ces relations sont en principe «couvertes» ou «celées» (p. 180) (concept important qui renvoie au fait que le «serviteur» ne doit pas, en principe, se déclarer). Parmi les thèmes abordés dans ce cadre, il y a celui de la mort du «serviteur» ou de la femme, parce que la censure de la pulsion sexuelle semble bien ne pas conduire à autre chose qu'à la mort, au mieux à la vie monastique. D'un autre côté, l'idée que le «service» de la femme aimée n'est qu'une conduite hypocrite ou provisoire, parfois impossible à maintenir jusqu'au bout (on va difficilement contre la nature), parfois destinée à dissimuler les véritables intentions du «serviteur», qui n'ont rien de chaste, apparaît assez souvent. Comme chez d'autres écrivains de l'époque (Ronsard, Du Bellay, Jodelle etc.) le sublime du pétrarquisme fait souvent l'objet d'une attaque en règle de la part d'Hircan notamment.

3. La religion est un troisième thème, traité de deux façons: d'une façon traditionnelle d'abord: les moines sont souvent paillards, violents, hypocrites, comme dans la littérature satirique du Moyen Age; d'autre part dans le cadre des débats d'actualité: l'idée souvent développée est que l'homme est pécheur et que l'idée de lui attribuer (de s'attribuer) des mérites procède d'un orgueil satanique; c'est la grâce divine, toujours imprévisible et gratuite, qui sauve et elle seule.

On notera que bien que l'œuvre puisse être lue comme une chronique du règne de François I ${ }^{\text {er }}$ (voir plus haut), le politique n'y apparaît presque jamais (nouvelle 17 exceptée), si ce n'est dans un éloge tellement emphatique, qu'il a une valeur psychologique plus que politique, du roi. 